\title{
EL PRINCIPIO DEL CIERRE CAUSAL DEL MUNDO FÍSICO*
}

Agustín Vicente

\author{
Departamento de Lógica, Historia y Filosofía de la Ciencia \\ Universidad de Barcelona \\ avicente@trivium.gh.ub.es
}

Quienes ven alguna diferencia entre alma y cuerpo es que carecen de ambos

Oscar Wilde

RESUMEN: Cabe argumentar en favor del fisicismo a partir de consideraciones metodológicas o epistémicas, o desde un punto de vista ontológico. En los últimos años se ha venido presentando un potente argumento ontológico que hace un uso esencial de lo que se ha dado en llamar el "principio del cierre causal del mundo físico". En este artículo examino si es posible que sea la propia física quien fundamente este principio. Propongo que, con la ayuda de las contemporáneas teorías reductivas de la causalidad a intercambio o transferencia de cantidades conservadas, las leyes de conservación pueden proporcionar tal fundamento. También evalúo qué fuerza modal puede tener este principio del cierre.

PALABRAS CLAVE: fisicismo, principio del cierre causal del mundo físico, leyes de conservación, cantidades conservadas

SUMMARY: It is possible to argue for physicalism from methodological or epistemic considerations or from an ontological position. In the last years one can find a powerful ontological argument for physicalism which makes essential use of what has been labeled "the principle of the causal closure of the physical world". In this paper I examine whether this principle can be grounded in physics itself. I propose that, with the aid of contemporary reductive transference or exchange theories of causation, conservation laws can provide such a basis to the principle of the causal closure. I also consider what modal force the principle may have.

KEY WORDS: physicalism, principle of the causal closure of the physical world, conservation laws, conserved quantities

* Agradezco la ayuda de Francisco Cano, y los comentarios de José Luis Falguera, Juan Vázquez y José Miguel Sagüillo, y especialmente los comentarios precisos y elegantes hechos por un evaluador anónimo de Crítica, que han sido decisivos para mejorar el artículo. La investigación para este artículo ha sido financiada por una beca posdoctoral del Gobierno Vasco y el proyecto UPV/EHU 109.109-HB078/99 de la Universidad del País Vasco. 


\section{Introducción}

Las discusiones acerca de la causación mental y el fisicismo giran a menudo alrededor de un principio que se asegura es dictado por la física contemporánea. Tal principio afirma que todo efecto físico (es decir, todo evento físico causado) tiene una causa completa igualmente física. Es decir, en la producción de un efecto físico cualquiera no tienen por qué intervenir entidades no físicas, ni siquiera como causas parciales. ${ }^{1}$ Este principio recibe el nombre de 'principio del cierre causal del mundo físico'.

Este principio del cierre causal provoca problemas de exclusión cuya única solución verosímil es la reducción. Cualquier evento, proceso u objeto que estimemos que produce efectos físicos ha de ser físico, pues, salvo en los intrincados casos de sobredeterminación causal, no puede haber dos causas completas para un mismo efecto. Este problema se aplica con la mayor intensidad a la relación cuerpo-mente: por una parte, afirmamos que nuestras creencias y deseos causan cambios en el mundo físico; por otra, el principio del cierre causal más las consideraciones anteriores acerca de la copresencia de causas dicta que tales cambios no tienen más que causas físicas. Conclusión fisicista: los eventos o procesos mentales son eventos o procesos físicos.

El fisicismo, no obstante, puede parecerle a muchos una postura extrema. De hecho, los intentos por evitar la conclusión reductivista han sido numerosos y variados. Ejemplos clásicos son el dualismo interaccionista o el emergentismo. Dejando de lado sus diferencias, ambas posturas coinciden en sostener que los eventos mentales, aun siendo esencialmente distintos de los eventos físicos, operan cambios en el mundo físico. Además, ambas afirman que el principio del cierre causal del mundo físico es falso. Otra postura antirreduccionista que merece interés es la

${ }^{1}$ El principio del cierre causal no afirma que todos los eventos físicos tengan causas físicas, pues de lo contrario iría en contra de la física (cuántica) contemporánea. Lo que afirma es que si un evento físico tiene una causa, ésta es física. Que la causa es completa quiere decir que engloba también a lo que suelen denominarse 'factores causales'. En definitiva, el principio del cierre sostiene que ninguno de los factores causales involucrados en la producción de un efecto físico es no físico. 
de la sobredeterminación. Según ésta (véase Hugh Mellor 1995, Tim Crane 1995), los eventos mentales tienen eficacia causal e inciden sobre un mundo que está causalmente cerrado, por lo tanto, están sobredeterminando sus efectos. De este modo, los defensores de la sobredeterminación son respetuosos con la verdad del principio del cierre causal del mundo físico, pero con ciertas restricciones modales. Rige en este mundo, pero existen mundos en los que los efectos aquí sobredeterminados son causados únicamente por los eventos mentales. ${ }^{2}$

Existen prima facie otras opciones de ser antifisicista que no necesitan ni de la falsedad ni de la contingencia del principio del cierre causal. Sin embargo, mi propósito es centrarme en las anteriores, o más bien, en el principio que niegan o acotan. Ya se mencionó que el principio del cierre causal es una verdad sostenida por la física contemporánea. Parece que, en efecto, esto es así. No obstante, no se trata de un principio físico; al menos, no figura en los libros de texto de física. Entonces cabe entender que tan sólo describe la aspiración de la física a ser explicativamente comprehensiva, aspiración que puede justificarse inductivamente. Alternativamente, puede considerarse que se trata de un principio que superviene sobre o se infiere de principios físicos, principios que sí figuran en los libros de texto de física. Mi propósito en este artículo es explorar esta segunda vía, que estimo más profunda, y evaluar, a la luz de lo que se descubra, la virtualidad de los antifisicismos considerados, i.e., dualismo interaccionista, emergentismo y sobredeterminación.

2 Decir que un evento $e$ está sobredeterminado causalmente quiere decir que existen dos causas $a$ y $b$ tal que: (1) si $a$ no hubiera causado $e$, lo habría hecho $b$, y (2) si $b$ no hubiera causado $e$, lo habría hecho $a$. Por lo tanto, (1') en todos los mundos cercanos en los que $a$ no causa $e, b$ lo causa, y (2') en todos los mundos cercanos en el que $b$ no causa $e, a$ lo hace. De este modo, tal como entiendo la tesis de sobredeterminación causal mente-cuerpo, decir que los eventos mentales y los eventos físicos sobredeterminan causalmente los movimientos corporales implica que existen mundos en los que los movimientos corporales no tienen una causa física, pero sí la tienen mental, i.e., el dualismo interaccionista y el emergentismo, aunque no son reales, son posibles. 


\section{Fundamentación del principio del cierre causal}

En realidad, la creencia en el principio del cierre causal del mundo físico es bastante antigua. $\mathrm{Al}$ parecer, los epicúreos sostenían un principio semejante, aplicado al mundo atómico de los cuerpos. Como muchos filósofos actuales, inferían la naturaleza material del alma a partir de su interacción con el mundo físico (cfr. Hamlyn 1973). Por lo tanto, cabe decir que la confianza en la existencia de una causa física para cada efecto físico es constante a lo largo de la historia y a través de las distintas ciencias físicas. Como digo, una explicación verosímil de este hecho consiste en incidir en el carácter básico de la física y en su potencial unificador. Intentamos explicar la mayor cantidad de hechos posible con la menor cantidad de instrumentos y entidades. Como corolario, tendemos a buscar las causas de los efectos básicos en ese mismo nivel fundamental. Además, esta tendencia se ve reforzada por el éxito, con lo que se convierte en firme creencia acerca de las relaciones causales en el mundo. ${ }^{3}$ De esta manera, el principio del cierre causal puede considerarse expresión del fisicismo, el mismo fisicismo que se supone se desprende del problema de exclusión que nuestro principio genera. No se puede decir que haya circularidad en esta forma de justificar y utilizar el principio del cierre causal, pero creo que la posición fisicista se hace más fuerte (e interesante) si, como dicen muchos filósofos, el principio del cierre causal tiene su justificación en la propia física.

Mi tesis es que son las leyes de conservación las que proporcionan, dentro de la física, un asiento al principio del cierre causal. Éstas, que a menudo son llamadas 'ideas guía', son verdades a posteriori y contingentes, aunque esto no quiere decir inmediatamente que nuestro principio haya de ser igualmente a posteriori y contingente (volveremos sobre esta cuestión más adelante). Se trata, de acuerdo con la física contemporánea, de leyes que rigen el comportamiento de la masa-energía, la carga

3 Ésta, que bien se puede llamar la 'vía metodológica' de fundamentación del principio, creo que se emparienta con el espíritu de nociones como la de "normas epistémicas y metodológicas" de Laudan, los "principios disciplinarios o ideales explicativos" de Toulmin, etcétera. 
y los momentos angular y lineal. Tales son las propiedades que la física hoy estima conservadas.

Algunos filósofos proponen reducir la relación de causalidad a una relación entre propiedades conservadas, o mejor, a una relación entre entidades (eventos o procesos: véase más adelante) cuyos objetos correspondientes instancian cantidades conservadas. De acuerdo con los distintos autores, tal relación puede ser de transferencia ( $c f r$. David Fair 1979), ${ }^{4}$ de transmisión ( $c f r$. Wesley Salmon) o simplemente de intercambio (cfr. Phil Dowe). ${ }^{5}$ Salmon (1994) introduce una segunda posible divergencia (que él mismo abandona en Salmon 1997) acerca de si las cantidades han de ser no sólo conservadas, sino también invariantes. El hacer uso de cantidades invariantes asegura que la relación de causalidad entre dos eventos o procesos particulares se perciba de igual forma en todos los marcos de referencia, pero tal vez eso no sea necesario. Basta con que la existencia de la relación sea invariante. La noción de causalidad admite cierta relatividad acerca de cuáles sean las causas y cuáles los efectos, aunque no acerca de si se ha producido un caso de causación o no. La reducción de la causalidad a la de un intercambio, transferencia, etc., de cantidades conservadas proporciona esa invariancia ( $c f r$. Fair 1979, p. 240; Dowe 1995, p. 333): los objetos cuyas líneas-mundo son causas o efectos (véase más adelante) instancian cantidades conservadas de un modo absoluto, aunque cuál sea la dirección del intercambio, o de la transferencia, dependa de los marcos de referencia.

También existen divergencias acerca de los relata ligados por la relación de causalidad. Mientras Fair intenta ser neutral acerca de las entidades relacionadas, y otros argumentan en favor de los eventos (cfr. Kistler 1997), Dowe y Salmon son partidarios de analizar la causalidad como una relación entre procesos o líneasmundo (una relación de intersección, para ser más precisos). La

4 Fair acota la relación a energía y momento, pero no parece que hubiera tenido ningún problema en incluir a la carga.

5 Estos términos, aparentemente sinónimos, tienen en realidad connotaciones muy diferentes dentro de las distintas teorías. Sin embargo, para los intereses de este artículo se pueden obviar tales diferencias. 
causación se convierte de este modo en un tipo de suceso físico peculiar, en el que dos procesos que se intersecan intercambian cantidades conservadas (o uno las transfiere al otro, etc.). En lo que sigue, dado que haré uso sobre todo de las ideas de Salmon y Dowe, adoptaré su individuación de los relata causales. Sin embargo, esta elección no tiene un peso decisivo en la argumentación, que ha de ser válida para cualesquiera otros relata.

Cada una de estas distintas teorías reductivas tiene sus pros y sus contras. Lo importante aquí, sin embargo, no son sus diferencias, sino lo que tienen en común, a saber, que la relación de causalidad es reducible a una relación física, y que depende de la existencia de leyes de conservación. Quizás sea excesivo sostener, como hacen los defensores de estas teorías, que la causalidad no es más que la transferencia, la transmisión o el intercambio de cantidades físicas conservadas. Como apunta David Fair, tal cosa implica que todos los relata de la causalidad son reducibles a la física, y ésta es una cuestión controvertida. Lo que sí es razonable sostener es que la causalidad en la física no es sino transferencia, transmisión o intercambio de cantidades conservadas. Tal vez otros ámbitos de la causalidad impliquen entidades diferentes (aunque, según nos dice el argumento de la exclusión, no si la relación se establece con efectos físicos) y la relación de causalidad consista en alguna otra cosa, o sea quizás irreducible. Lo que sí parece cierto es que cuando la relación se da entre eventos, objetos o procesos físicos, lo que hay es una transferencia, transmisión o intercambio de cantidades conservadas.

Para fundamentar el principio del cierre causal en las leyes de conservación es necesario que quepa identificar de este modo efectos y causas físicas con cambios en cantidades conservadas. ¿Consisten los efectos físicos, todos, en cambios en cantidades conservadas? ¿No puede haber en física una relación causal que no consista en la transferencia, transmisión o intercambio de cantidades conservadas?

La respuesta negativa a esta pregunta se fundamenta en la distinción entre procesos y seudoprocesos, en la que ha trabajado intensamente Wesley Salmon. Antes de apostar por la teoría de transmisión de cantidades conservadas, Salmon (cfr. 1984) sostuvo durante largo tiempo una teoría en la que la causalidad 
se analizaba en términos de la transmisión de marcas. Tanto entonces como ahora, el principal objetivo de Salmon ha sido distinguir entre procesos causales y seudoprocesos. ${ }^{6}$ En su antigua teoría, se definía (i) un proceso como algo que muestra una consistencia de características, (ii) una marca como la alteración de una característica que acontece en una única intersección local, (iii) un proceso causal como un proceso capaz de transmitir marcas, y (iv) una interacción causal como la intersección de dos procesos en la que ambos resultan permanentemente marcados. Con este aparato se intentaba trazar una línea de demarcación entre procesos causales y procesos tales como el movimiento de una sombra. El caso más discutido por Salmon tiene como protagonista a un emisor de luz que rota en el centro de un edificio circular. Una breve emisión de luz que viaja del foco a la pared es un proceso causal. Si se coloca un filtro rojo en su camino, el haz se vuelve rojo, y permanece rojo desde el punto en que se ha cruzado con el filtro hasta la pared, sin ninguna intervención ulterior. En contraste, el punto de luz que recorre la pared es un seudoproceso. Se puede tornar rojo por breves instantes si, por ejemplo, se coloca un filtro en un punto de impacto en la pared, pero pasado ese punto dejará de ser rojo.

La teoría de las cantidades conservadas coincide en este diagnóstico general. Procesos tales como el movimiento de una sombra o el del punto de luz del ejemplo de Salmon son seudoprocesos. El punto de luz, en su movimiento a lo largo de la pared, posee velocidad y luminosidad, pero no tiene energía, ni momento ni carga. Éstos son privilegio exclusivo del haz de luz y de la pared sobre la que impacta (véase Dowe 1992). El difuso concepto de marca adquiere de esta forma un significado preciso: las marcas son cambios en cantidades conservadas. En este senti-

${ }^{6}$ Una distinción que les resulta prima facie difícil de trazar a regularistas y defensores del análisis en contrafácticos. La "limpieza" con que acomodan nuestras intuiciones las teorías de Dowe y Salmon es, creo, el argumento más poderoso en su favor. No obstante, no es mi intención entrar en un debate "serio" con regularistas y contrafactualistas, que quizás puedan llegar a ofrecer una (buena pero a buen seguro compleja) reinterpretación de las teorías de Salmon y Dowe. El que lo puedan hacer, en cualquier caso, no afecta las tesis aquí defendidas. 
do, la teoría de las cantidades conservadas supone una mejora de la teoría inicial de las marcas: coincide en sus diagnósticos (que a su vez coinciden con los de la intuición), pero es más precisa y profunda. ${ }^{7}$ De hecho, puede explicar por qué el movimiento de una sombra o el del punto de luz en el edificio circular no son procesos causales. Magnitudes como la velocidad no pueden ser transferidas, o intercambiadas, porque no se conservan. En un choque "clásico" (i.e., elástico, inelástico o plástico) el momento incidente es igual al saliente, y cabe decir de este modo que el objeto incidente transfiere su momento al saliente, o que ambos intercambian sus momentos. Pero nada semejante se puede decir acerca de la velocidad: la velocidad entrante puede desaparecer en gran parte. Se explica así que un proceso línea-mundo de un objeto que posee velocidad, pero no masa, no pueda ser un proceso causal. Para contar como proceso causal, el objeto ha de poseer alguna propiedad transferible, o intercambiable, y esto quiere decir alguna propiedad conservada.

Estas consideraciones suponen una fuerte motivación para la teoría adoptada, esto es, que algo (evento o proceso) puede ser una causa o un efecto si y sólo si el objeto correspondiente instancia una cantidad conservada. ${ }^{8}$ Cabría protestar y aducir que el movimiento de una sombra, o el del punto de luz en el edificio circular, son efectos físicos, resultados de la operación de causas físicas. Pero estos movimientos son, a lo sumo, epifenómenos, y, de acuerdo con principios verosímiles, un evento, proceso o propiedad que no tenga poderes causales no puede encontrar un sitio en la ontología.

7 Otra gran ventaja que Salmon encuentra (véase Salmon 1994) es que la teoría de las cantidades conservadas le permite prescindir de un remanente del análisis en contrafácticos con que cargaba su teoría de la transmisión de marcas.

${ }^{8}$ En el caso de los eventos, al menos en el caso de los eventos kimianos, por 'el objeto correspondiente' se entiende el objeto que individualiza al evento (un evento kimiano es la instanciación, por parte de un objeto, de una propiedad en un tiempo determinado). En el caso de los procesos, el objeto correspondiente es aquel objeto cuya línea-mundo es el proceso en cuestión (un proceso es la línea-mundo de un objeto; un proceso causal, por tanto, es la línea-mundo de un objeto que instancia una propiedad conservada). 
Nótese que la argumentación anterior no incurre en una petición de principios. Lo que trata de mostrar es que los efectos físicos son, o implican esencialmente, variaciones en los valores de las propiedades conservadas instanciadas por los objetos de ciertos eventos o procesos. Del mismo modo, se trata de proporcionar un argumento en favor de la teoría de las cantidades conservadas. Por lo tanto, no se puede hacer uso de la propia teoría. Se incurriría en una petición de principios, de este modo, si se argumentara de la siguiente manera: (i) sólo pueden ser causas las entidades (eventos o procesos) cuyos objetos correspondientes instancian cantidades conservadas; (ii) (de (i)) los efectos cuyos objetos correspondientes instancian otras propiedades físicas, y no propiedades conservadas, no pueden ser causas; (iii) (de (ii)) tales efectos son epifenómenos y no encuentran asiento en nuestra ontología.

Para llegar a esta conclusión es necesario utilizar hechos o argumentos externos a la teoría de las cantidades conservadas. Existen otras razones para creer en esta teoría, pero la que se ha proporcionado aquí es que se adecua al diagnóstico que la intuición, y la anterior teoría de Salmon como expresión elaborada de ésta, emite acerca de casos como el del movimiento de una sombra o el del punto de luz. Estimamos estos casos independientemente como ejemplos de seudoprocesos o epifenómenos. La teoría de las cantidades conservadas coincide con esta estimación intuitiva. Aparte, proporciona su propia explicación: los eventos o procesos cuyos objetos correspondientes instancian velocidad, si no instancian también masa, carecen de poderes causales, ya que la velocidad es una magnitud no transferible o intercambiable. Se trata, por lo tanto, de una teoría que cubre los hechos que le corresponden con un plus de poder explicativo e inteligibilidad respecto de otras. Creo que ésta es la principal razón aducible en su favor.

\section{Estatuto modal del principio del cierre causal}

Tenemos entonces ya la fundamentación física del principio del cierre causal. Tal y como se ha presentado, su verdad depende de la verdad de la reducción de la causalidad a una relación de 
transferencia o intercambio de cantidades conservadas. Y ésta, a su vez, depende de la existencia de leyes de conservación.

No es, quizás, la única vía de fundamentación. Algunos filósofos prefieren reducir la causalidad a la acción de fuerzas (e.g. Heathcote 1989, Bigelow y Pargetter 1990a). Una reducción de este cariz (limitada, de nuevo, a la física) puede proporcionar un argumento distinto para el principio del cierre causal, cuya validez dependería de poder limitar las fuerzas actuantes sobre los fenómenos físicos a fuerzas físicas, esto es, a la fuerza electrodébil, la fuerte y la gravitatoria, o, eventualmente, a una única fuerza. ${ }^{9}$ No parece que sea en exceso complicado, pero es una vía que no exploraré. Por un lado, las teorías de las cantidades conservadas están más desarrolladas y son más convincentes; por el otro, las leyes de conservación son las leyes más fundamentales de la física, mientras que las fuerzas tienen un estatuto derivado.

Tal y como se ha dicho al principio, las leyes de conservación son leyes a posteriori. ${ }^{10}$ Tómese el caso de la primera ley de la termodinámica, la ley de la conservación de la energía. Durante largo tiempo se creyó que la energía mecánica ejercida en contra de fuerzas de fricción era energía perdida, hasta que en el siglo XIX, especialmente gracias a los experimentos de Joule (1844, 1845), se descubrió que el calor era una forma de energía. Hasta entonces se había pensado que la energía se conservaba sólo en sistemas privilegiados y que no era un principio de aplicación

9 A modo de curiosidad, cabe comentar que un argumento semejante es el que utilizaba Carl Sagan en contra de la astrología. Su idea es que la física ha probado que sólo hay tres fuerzas que actúen en el mundo físico y ninguna de ellas media la interacción entre la posición de Venus y el comportamiento de los seres humanos en el sentido que la astrología sugiere.

10 En este trabajo no estoy especialmente interesado en el estatuto epistémico de las leyes de conservación, sino en su estatuto modal (metafísico). Hay filósofos, por ejemplo Shoemaker, que defienden que todas las leyes son necesarias. Si así lo fueran, entonces también las leyes de conservación serían necesarias, la reducción de la causalidad física al intercambio, etc., de propiedades conservadas también, y, por lo tanto, nuestro principio sería igualmente necesario y el dualismo imposible. Pero si no lo son - y supondré a partir de este momento, por mor de la argumentación, que no lo son-, entonces es razonable pensar que una ley será necesaria sólo si es una verdad conceptual. Por esta razón dedico este apartado a mostrar que las leyes de conservación no son conceptualmente verdaderas. 
universal, en parte debido a la doctrina química de que el calor era una sustancia indestructible. Pero los experimentos de Joule probaron que calor y energía eran interconvertibles, y con ello, que era razonable considerar que el principio de conservación era verdadero universalmente.

El siguiente hito experimental en la historia del principio de conservación de la energía tuvo lugar en los años veinte y treinta, en el contexto del descubrimiento y posterior estudio de la desintegración beta. Tal desintegración tenía un rasgo muy peculiar: los electrones emitidos en ella - partículas beta - mostraban una distribución continua de su energía en el espectro, desde casi cero a valores bastante altos. Sin embargo, el núcleo perdía energía sólo en valores discretos, que por lo general eran bastante mayores que el valor de la energía que se llevaban consigo las partículas beta. Niels Bohr propuso explicar este fenómeno como una violación de la ley de la conservación de la energía: según él, en algunos casos de desintegración beta, la energía no se conservaba. Pero físicos más cautos, como Pauli y Fermi prefirieron postular la existencia de partículas sin carga y casi sin masa que se emitían junto con las partículas beta en la desintegración, y que se llevaban parte de la energía perdida por el núcleo: los neutrinos. Éstos fueron finalmente "capturados" en los años cincuenta, y, de nuevo, la ley de la conservación de la energía recibió refrendo experimental.

Por otra parte, hay principios de conservación que han resultado ser falsos. El caso más espectacular es el del principio de conservación de la paridad, pero no es el único. Por ejemplo, en la interacción fuerte entre neutrones y protones se conservan, aparte de la paridad, magnitudes tales como la masa llamada bariónica (número total de protones y neutrones), el isospín y la hipercarga, que no se conservan en la interacción electrodébil. Podría haber ocurrido que, conociendo mejor la interacción fuerte que la electromagnética y la débil, estas magnitudes se hubieran postulado como universalmente conservadas. En un caso así, el descubrimiento de las nuevas interacciones habría supuesto una refutación empírica de las supuestas leyes de conservación.

No cabe duda de que los principios de conservación de la energía, los momentos lineal y angular y la carga están fuerte- 
mente atrincherados en las creencias de los físicos, y no son por lo tanto fácilmente revisables a la luz de la evidencia empírica. Pero eso no quiere decir ni mucho menos que sean a priori. Que no sean a priori, por otra parte, quiere decir, a mi entender, que carecemos de una razón de principio para considerarlos metafísicamente necesarios. Si existe espacio para una distinción entre la modalidad física y la metafísica (véase la nota 10 ), entonces parece que hemos de decir que hay mundos cuya física no está gobernada por nuestras leyes de conservación.

Según esto, ¿qué estatuto habremos de otorgar al principio del cierre causal del mundo físico? La respuesta no es directa, ni sencilla. En efecto, dado que, en el argumento presentado, el principio del cierre causal depende en primera instancia de la reducción de la relación causal a una de intercambio o transferencia de cantidades conservadas, su estatuto depende más del de esta reducción que del de las leyes de conservación. Todos los defensores de las teorías de las cantidades conservadas (así como los proponentes de la reducción a fuerzas) coinciden en sostener que la identificación es a posteriori. Sin embargo, no existe la misma uniformidad en su evaluación modal. Para algunos (e.g. Bigelow y Pargetter 1990a, Kistler 1997), la reducción es necesaria; para otros ( $c f r$. Salmon 1984, Dowe 2000), se trata de una identidad contingente.

Que la reducción sea necesaria quiere decir que en todos aquellos mundos en los que no rigen nuestras leyes de conservación, tampoco hay causalidad (física, al menos). Por consiguiente, el principio del cierre causal sería trivialmente falso en esos mundos: no todos los eventos físicos causados tienen causas físicas porque ninguno las tiene. Si entendemos, como en principio, he propuesto que la reducción a intercambio, etc., de cantidades conservadas sólo alcanza a la causación física, entonces en esos mundos carentes de leyes de conservación, el dualismo interaccionista puede ser un hecho, ya que la causalidad no física pervive. Esto, en principio, quiere decir que el dualismo es considerado en nuestro mundo como una posibilidad, y, por lo tanto, que no puede descartarse que nuestros movimientos corporales estén aquí sobredeterminados causalmente. Es más, en esos mundos en los que los eventos mentales (y, en general, 
los eventos de aquellos dominios de los que cabe postular un dualismo) interactúan causalmente con eventos físicos, los primeros son la única causa posible de los segundos. No obstante, dado que no sabemos en qué puede consistir la causalidad en otros ámbitos, y, sobre todo, ignoramos cómo esos otros ámbitos pueden relacionarse causalmente con el mundo físico, creo que lo más oportuno es declararse agnóstico (véase más adelante) acerca de las posibilidades consideradas.

Por otra parte, si la reducción alcanza a todo tipo de relación causal, tal y como sus defensores proponen, entonces el principio del cierre causal resultaría necesariamente verdadero, aunque su rango de aplicación sería muy limitado. El emergentismo y el dualismo serían imposibles, y la sobredeterminación igual de quimérica.

Finalmente, si adoptamos una visión más relajada sobre la causalidad, en particular, si consideramos 'causalidad' y términos similares como términos no rígidos, los resultados pueden bien ser otros. La causalidad (física) en este mundo se identifica con el intercambio o transferencia de las cantidades aquí conservadas, pero en otros mundos puede consistir en otra cosa. Dowe (2000) sostiene que su teoría es agnóstica incluso para aquellos mundos en los que se conservan otras cantidades, distintas de las conservadas aquí. Es decir, las predicciones modales de la teoría son casi nulas. En cuanto a los mundos en los que no hay leyes de conservación, presumiblemente todo depende de cuál sea el significado de 'causalidad' y de los términos de esa familia. En su artículo prekripkeano, Fair (1979, p. 232) afirma que "si el análisis mecánico estadístico de la temperatura no puede ser culpado por no decirnos qué es la temperatura en todos los mundos posibles, el análisis fisicista de la causación debería compartir la inmunidad del análisis a tal crítica". En el apogeo de las identidades fisicistas necesarias y a posteriori en que nos encontramos hoy en día, esta posición no parece fácil de defender. La analogía, desde luego, es condenatoria. Pero quizás, como digo, pueda sostenerse que, a diferencia de 'temperatura', 'causalidad' no es rígido.

Si esto fuera posible, ¿qué ocurriría con el principio del cierre causal? Es decir, si en principio es posible que haya causalidad 
física incluso allí donde no existen leyes de conservación, ¿puede haber en esos mundos efectos físicos sin causa física? Depende de si en estos mundos es posible dar con una reducción fisicista de la causalidad tal que ésta ligue sólo entidades físicas. Si Dowe está en lo cierto, ésta es una cuestión sobre la que no podemos tener una idea razonable. No sabemos lo suficiente ni de la física en esos otros mundos ni de la noción de causalidad. En otras palabras, nada de lo que hemos dicho nos permite descartar que en un mundo sin leyes de conservación haya causación mental, o causación emergente, aunque tampoco nos permite afirmarlo. No cabe afirmar, entonces, que haya mundos en los que la relación de causalidad, aquí realizada por el intercambio o transferencia de propiedades conservadas, se realice, en ocasiones, por la acción de propiedades mentales sobre el mundo físico. Más bien, sólo es "concebible" en un sentido nada preciso. ${ }^{11}$

Creo que este agnosticismo modal es coherente, razonable y, en este caso, recomendable. Su consecuencia es que hablar del alcance modal del principio del cierre causal más allá de los mundos físicamente posibles (en sentido restringido), y tras ello, del dualismo interaccionista, el emergentismo y la sobredeterminación, es pura especulación.

11 Bigelow y Pargetter, (1990b), analizan la noción de ser concebible como ser posible que sea posible, i.e., $p$ es concebible si existe un mundo posible $m_{1}$ accesible desde el nuestro tal que desde ese mundo se puede acceder a otro mundo $m_{2}$ (inaccesible para nosotros) en el que $p$ es un hecho. Si ésta fuera la noción de concebibilidad involucrada, entonces la sobredeterminación causal mente-cuerpo sería una posibilidad. Esto es, ha de haber un mundo posible, no el real, en el que haya causas mentales para algunos efectos físicos que ya tienen su propia causa física. Dado que la tesis de sobredeterminación consiste en la postulación de mundos posibles en los que el dualismo interaccionista es cierto, si decimos que éste sólo es posible que sea posible, entonces no puede darse sobredeterminación en nuestro mundo. Pero se dará en un mundo desde el que el dualismo se considere como una posibilidad, esto es, en uno de esos mundos que nosotros mismos consideramos como posibles. No obstante, como digo, creo que el sentido de concebibilidad que, siguiendo a Dowe, podemos manejar no es éste. 


\section{BIBLIOGRAFÍA}

Bigelow y Pargetter, 1990a, "Metaphysics of Causation", Erkenntnis, 33, pp. 89-119.

, 1990b, Science and Necessity, Cambridge University Press, Cambridge.

Crane, Tim, 1995, "Mental Causation", Proceedings of the Aristotelian Society, vol. 69, pp. 211-236.

Dowe, Phil, 2000, "The Conserved Quantity Theory Defended", Theoria, 15, pp. 11-31.

— 1995, "Causality and Conserved Quantities: A Reply to Salmon", Philosophy of Science, 62, pp. 321-333.

— , 1992, "Wesley Salmon's Process Theory of Causality and the Conserved Quantity Theory", Philosophy of Science, 59, pp. 195216.

Fair, David, 1979, "Causation and the Flow of Energy", Erkenntnis, 14, pp. 219-250.

Hamlyn, D.W., 1973, "La filosofía griega posterior a Aristóteles", en D.J. O'Connor (comp.), Historia crítica de la filosofía occidental: la filosofía en la Antigüedad, Paidós, Buenos Aires.

Heathcote, Adrian, 1989, “A Theory of Causality: Causality = Interaction (as Defined by a Suitable Quantum Field Theory)", Erkenntnis, 31, pp. 77-108.

Kistler, Max, 1997, "Reducing Causality to Transmission", Erkenntnis, 48, pp. 1-24.

Mellor, Hugh, 1995, The Facts of Causation, Routledge, Londres.

Salmon, Wesley, 1997, "Causality and Explanation: A Reply to Two Critiques", Philosophy of Science, 64, pp. 461-477.

— 1994, "Causality Without Counterfactuals", Philosophy of Science, 61, pp. 297-312.

—_ 1984, Scientific Explanation and the Causal Structure of the World, Princeton University Press, Princeton.

Recibido el 20 de marzo de 2001; revisado el 31 de agosto de 2001; aceptado el 5 de septiembre de 2001 